\title{
Gold - Section in Interactive User Interface Development
}

\author{
Uldis Žaimis'1, Ilva Magazeina², Anita Jansone ${ }^{3}$ \\ Faculty of Science and Engineering, University of Liepaja \\ Address: Valdemara 4, Liepaja, LV3401, Latvia \\ ${ }^{1}$ uldis.zaimis@liepu.lv, ${ }^{2}$ ilva.magazeina@liepu.lv, ${ }^{3}$ anita.jansone@liepu.lv
}

\begin{abstract}
The days when only specialists worked with programs and the functionality was the most important have long passed. In today's competitive environment, an important marketing component is reliability in which the only tangible part of the program, the interface, plays a large role. Purely psychological, it is important for the users' visibility, correctness and can even be argued, friendliness. This is determined by the elements of visibility and components' location in intuitively expected places, design integrity, tone and choice of characteristic elements which, together, create a feeling of sympathy or antipathy from the software user. Regularities, which ultimately determine the user's subconscious reactions, are found in natural formations - from the most basic proportions of plants to more sophisticated self-arranged compositions.

The harmony of the structures of natural systems, that is, their internal organization, is subject to certain mathematical laws. Objective world stable stationary states corresponding to particular figures, called generalized Golen Ratios. These figures are all the structure of the invariant, which are embodied by the dialectic structure of the world and the different variations that can be observed at every step of nature. It is important to note that with the generalized Golden Ratio, not only is the well-known ratio of 1:1,618 understood, but a whole line of relationships, where like in music, a single major or minor note can be played, and another can stand out from the whole ensemble.

The main applications of the Goden Ratio in interface design are space division, caption-font size ratios, restrictive areas (buttons), title queue length, color tone saturation ratio, and cell location coordinates.

In this paper, we propose certain recomendations for the development of a user - friendly interface. These recommendations suggest uses in the software developer training process for non-design specialty students; target group programmers, computer specialists, and IT project managers. The article does not address the development of graphic design tools and their functionality.
\end{abstract}

Keywords: golden ratio, design, interface, computer software.

\section{INTRODUCTION}

An important role in modern software development is making an easy, readable user interface. Occasionally, its attractiveness is the exact cause of a potential customer's choice in favor of one or other competing software products. As with any visual design object, the interface has parallel functional properties - input / output, the operations to be carried out and explanatory elements. It also has a purely decorative property, a design that has a unified style that generally is attractive and gives an easy readable feeling to the user.

The days when only specialists worked with programs and the functionality was the most important have long passed. In today's competitive environment, an important marketing component is reliability in which the only tangible part of the program, the interface, plays a large role. Purely psychological, it is important for the users' visibility, correctness and can even be argued, friendliness. This is determined by the elements of visibility and components' location in intuitively expected places, design integrity, tone and choice of characteristic elements which, together, create a feeling of sympathy or antipathy from the software user. Regularities, which ultimately determine the user's subconscious reactions, are found in natural formations - from the most basic proportions of plants to more sophisticated self-arranged compositions.

The main applications of the Golden Ratio in interface design are space division, caption font size ratio restrictive areas (buttons), filling the title queue length, color tone saturation ratio, and cell location coordinates.

\section{MATERIALS AND METHODS}

Golden Ratio- historical size/value ratio

The Golden Ratio, or "split of harmonious parts" has been known from antiquity. It has its origins in the written form associated with Euclid (around 300 $\mathrm{BC}$ ), however, proof has been found Pythagoras (6th century BC) and even earlier authors [1], [2] used it. A real boom to find relationships began around the 15 th century, when they were used in art (see. Figure $1)$, and architecture. 


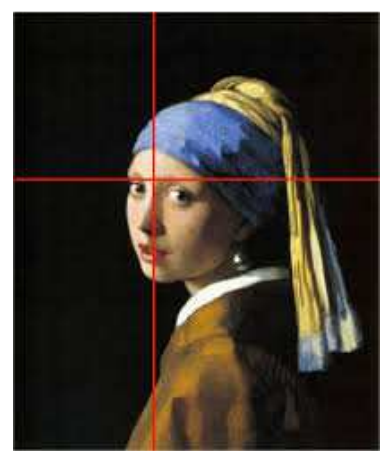

Figure 1. J. Vermeer "Girl with a pearl earring", 1665

\section{Generalized Golden Ratio}

The harmony of the structures of natural systems, that is, their internal organization, is subject to certain mathematical laws. Objective world stable stationary states corresponding to particular figures, called generalized gold sections. These figures are all the structure of the invariant, which are embodied by the dialectic structure of the world and the different variations that can be observed at every step of nature. It is important to note that with the generalized Golden Ratio, not only is the well-known ratio of 0.618 understood, but a whole line of relationships, where like in music, a single major or minor note can be played, and another can stand out from the whole ensemble.

The generalized Golden Ratio can be calculated according to the following scheme. Denote the probability of the event with $p$, in which case, the complement up to 1 will be $(1-p)$, describing the event default probability. To express the amount of information, we take the logarithms of the above values, and denote the relationship of this logarithms with $k$ :

$$
k=\frac{\log (1-p)}{\log p}
$$

where from,

$$
\log (1-p)=k \log p
$$

which in turn, we obtain [3]:

$$
p^{k}+p-1=0
$$

The set of resulting solutions of the equation is shown in Table 1.

Table 1.

Generalized Golden Ratio Examples

\begin{tabular}{ccc}
\hline $\mathrm{k}$ & $\mathrm{p}$ & $1-\mathrm{p}$ \\
\hline 1 & 0,5000 & 0,5000 \\
2 & 0,6180 & 0,3820 \\
3 & 0,6823 & 0,3177 \\
4 & 0,7245 & 0,2755 \\
5 & 0,7549 & 0,2451 \\
6 & 0,7781 & 0,2219 \\
7 & 0,7965 & 0,2035 \\
\hline
\end{tabular}

\begin{tabular}{ccc}
\hline 9 & 0,8243 & 0,1757 \\
10 & 0,8351 & 0,1649 \\
11 & 0,8444 & 0,1556 \\
12 & 0,8525 & 0,1475 \\
\hline
\end{tabular}

The following table shows the different ratios with values rising at an exponential rate.

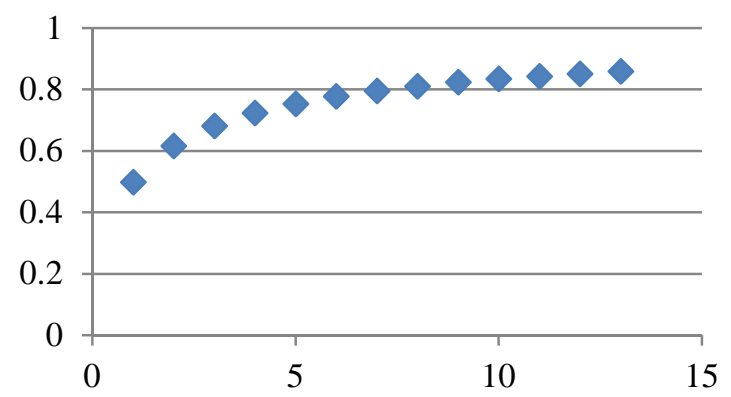

Table 2. Generalized Golden Ratio display

Other generalized Golden Ratio detection methods can be found in the literature [4], [5].

Historically, the ratio applied to $k=2$ has been often used in architecture and art, and found in living organisms and other natural formations proportions [3], [5]. There is a widespread perception that it exactly mathematically expresses the harmony of natural formations.

Higher-level (rank) proportions, whose conformity with nature's harmony gradually decreases [3], shall be used if the number of cells, their role in the situation, or other considerations does not allow the use of commonly known ratio of 1 : 1.618 .

\section{Generalized Golden Ratio applications in user interfaces}

Graphic user interfaces (GUI) can include the following applications of the generalized Golden Ratio:

- size of an object relative to the total or boundary area,

- object aspect ratio,

- small object, logo, or buttons centre position coordinates,

- phrase and title length ratio (with one or more rows) in relation to the body of the text,

- font size of the different meanings (importance) in the text,

- ratios of colour saturation - both grouping objects (e.g., push-buttons or web-addresses) according to their meaning and, in particular, marking watched/attended; this same ratio can be used when the colour area should be limited on the same tone, but richer colour line.

Mainly it is used to understand the user interface for computer display (screen). But in recent years, 
there has been an explosion of smartphone and tablet development. These devices are increasingly being used for games, education, and management of various processes (directly and with the help of Bluetooth technology). Here, too, object placement, size, and proportion design can apply the generalized Golden Ratio (example Fig. 2.).

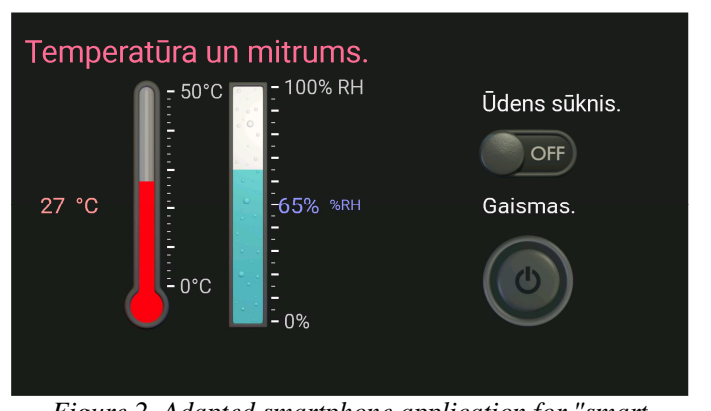

Figure 2. Adapted smartphone application for "smart greenhouses" management. Smartphone screenshot. Author A. Lastenieks.

Some possible inconveniences for the development of smartphones and tablet interfaces are many blanks and that the application does not support the free object layout, but only with fixed steps due to the limited size of the position. Because of their nature, development of smartphones and tablet interfaces require further research.

\section{RESULTS AND DISCUSSION}

Object position and object size ratio are two of the most visible user interface parameters. These can take the forms of square aspect ratio, space, object coordinates in both the vertical and horizontal directions, and distances between the centres of objects.

Modern web-pages are often designed in the form of scrollable columns; in this case, the presentation of the composition forms the column widths. Figure 3 shows a traditional column split with a ratio of 1 : 1.62 .

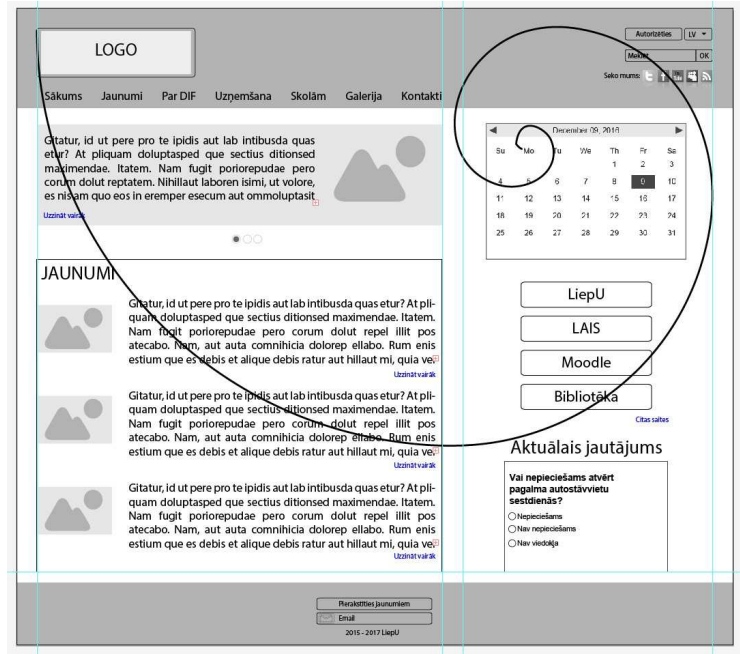

Figure 3. Column split ratio 1:1,62.
It can be seen that the separation is not optimal, because the right-hand column is relatively free with respect to the left-hand column. The page itself (prototype) with split ratio of 1: 1.32 can be seen in Figure 4.

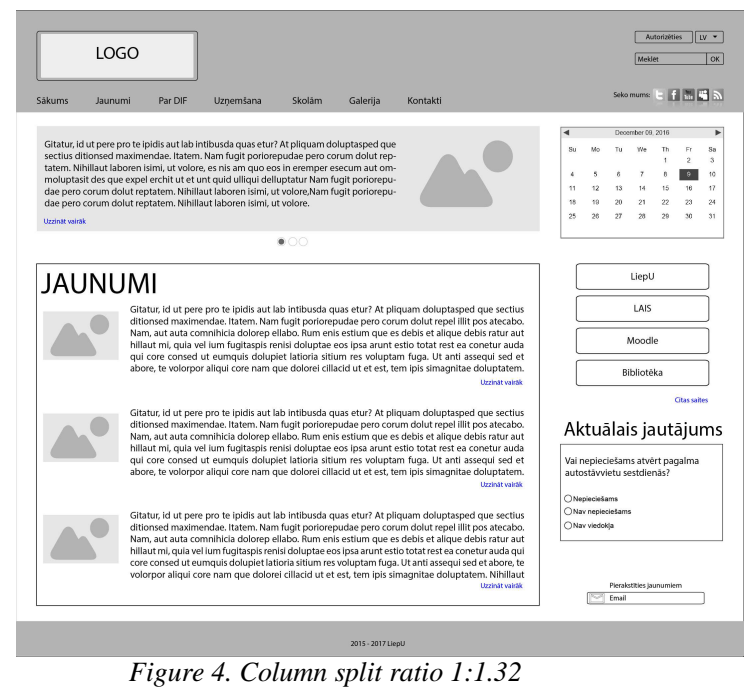

By contrast, in a multi-column page, it is possible to use mixed divisions where the size of each column down by the generalized golden ratio relationship. Of course, there must first be guided by the amount of information to display along with conceptual and textual meanings (example in Figure 5).

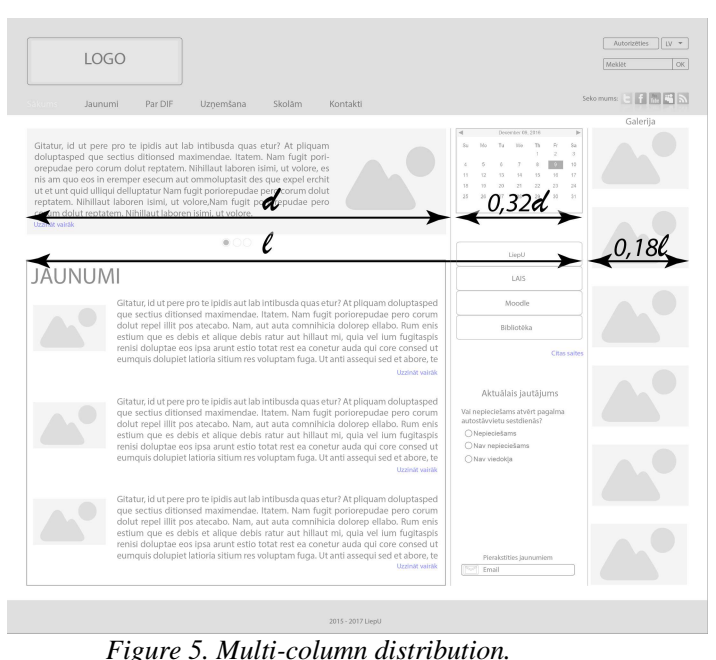

Colour saturation control is easy to use when working with graphic editors; in which case this parameter is expressed numerically and is easily calculable. The authors do not provide recommendations for keynote colour choice; they are determined by completely different considerations. But often there are cases when the base colour will be applied at different saturation levels (for example, to distinguish a once visited the website address or a pressed push-button). Unfortunately, this paper is 
printed in black and white (greyscale) format and therefore, colourful examples are not provided.

The ratio of font sizes between the main body and heading will rarely be used in documents whose meaning are determined style or even require certain conditions are met. Generalized Golden Ratio use can rather be found in letters of a private nature or artistic works. Font size is closely related to the title of the presentation. Also, in cases where the title font size is limited, the author's text should have a layout of one or more rows to make it one of the generalized Golden Ratio relationships, preferably one in which the overall scene is dominated by the given object. For example, a ratio of 1: 1.22 corresponds to the title of this article.

\section{CONCLUSION}

The application of the Generalized Golden Ratio in the development of user interfaces can significantly improve the readability and attractiveness of the products. Secondly, it facilitates the use of the developer's work. Since it is no longer enough that a regular object layout is formed with performance criteria and it is known that often interface developers are not professional designers, learning about the use of the Generalized Golden Ratio can serve as waypoints and assist with the finished presentation. Third, the use of the Generalized Golden Ratio in user interface development can and should be combined with other well-known aspects, such as the base colour corresponding to the product's development theme (business, education, cooking, games, etc.), sequential placement in the active field (confirmation button below or next to on the right of the previously entered information), etc. [6].

We recommend using the following steps for the development of graphical user interfaces:
- identify the functionally necessary interface objects: push buttons, titles, names, links, logos, etc.;

- develop an object grouping principle by the functionality or logical affiliation;

- work out interface areas: in the case of the absence of any other considerations (customer requirements, company documentation, unified style, regulatory provisions, etc.), select the split ratio by the generalized golden section scale, giving priority to the relationship with a lower sequence number (beginning with $\mathrm{k}=2$ ) while maintaining a balanced filling density; so the same principles apply different meanings in the text font size, button size and finer object positional relations;

- the changing of the title and text font sizes will necessitate returning to the preceding paragraph.

\section{REFERENCES}

[1.] Бендукидзе А. Д. Золотое сечение, «Квант» № 8, 1973

[2.] Roger Herz-Fischler. A Mathematical History of the Golden Number. Courier Corporation, 2013. ISBN 9780486152325

[3.] Сороко Э. М. Золотые сечения, прочессы самоорганизации и эволюции систем: Введение в общую теорию гармонии систем. Изд. 4-е. - М.: Книжньй дом

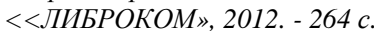

[4.] Falbo Clement E., Generalizations of the Golden Ratio, Sonoma State University, https://www.sonoma.edu/math/faculty/falbo/cmj123-134

[5.] Коновалов А.А. Обобщенные золотые сечения и их проявления в природных системах, https://www.trinitas.ru/rus/doc/0232/013a/2030-kn.pdf

[6.] Pressman Roger S. (2010) Software Engineering. A practitioner's approach. Seventh edition. McGraw-Hill, Pp. 312-346 